\title{
Nine theorems on the unification of quantum mechanics and relativity
}

\author{
A. Kryukov \\ Department of Mathematics, University of Wisconsin Colleges, 780 Regent Street, Madison, WI 53708
}

A mathematical framework that unifies the standard formalisms of special relativity and quantum mechanics is proposed. For this a Hilbert space $H$ of functions of four variables $\mathbf{x}, t$ furnished with an additional indefinite inner product invariant under Poincaré transformations is introduced. For a class of functions in $H$ that are well localized in the time variable the usual formalism of nonrelativistic quantum mechanics is derived. In particular, the interference in time for these functions is suppressed; a motion in $H$ becomes the usual Shrödinger evolution with $t$ as a parameter. The relativistic invariance of the construction is proved. The usual theory of relativity on Minkowski space-time is shown to be "isometrically and equivariantly embedded" into $H$. That is, classical space-time is isometrically embedded into $H$, Poincaré transformations have unique extensions to isomorphisms of $H$ and the embedding commutes with Poincaré transformations.

\section{INTRODUCTION}

It is commonly accepted that the improper states, such as eigenstates of position and momentum operators, cannot be elements of the Hilbert space of quantum states. Even though position and momentum operators are the building blocks of the theory, their eigenstates are not included in the formalism on an equal footing with the square-integrable states. Instead, the common rigorous treatment of such states uses the rigged Hilbert space construction of Gelfand Ref.[1], in which these states have no norm. At the same time, Hilbert spaces containing non-square integrable functions (on Lebesgue measure) are well known in mathematics. Moreover, some of these spaces are "close" to the standard spaces of Lebesgue square-integrable functions in the sense that they include the latter functions while practically preserving their norm. Perhaps the most obvious example is the Hilbert space $L_{2}(\mathbb{R}, \mu)$ of functions on $\mathbb{R}$ that are square-integrable with respect to the Gaussian weight $\mu_{L}(x)=e^{-\frac{x^{2}}{2 L^{2}}}$. Such a space contains the plane wave functions $e^{i p x}$. By applying the Fourier transform to $L_{2}(\mathbb{R}, \mu)$ one obtains a Hilbert space with the inner product

given by $\frac{L}{\sqrt{2 \pi}} \int e^{-\frac{L^{2}}{2}(x-y)^{2}} f(x) \bar{g}(y) d x d y$. Such a space contains the delta functions $\delta_{a}(x)=\delta(x-a)$. Moreover, as the parameter $L$ increases, the norm of any given square-integrable function $f \in L_{2}(\mathbb{R})$ in either of the above two metrics approaches the usual $L_{2}(\mathbb{R})$-norm of $f$ (see theorem 2).

Hilbert spaces "deformed" in such a way fit nicely into a general scheme in which an invariant abstract Hilbert space of states $\mathbf{S}$ has various realizations by Hilbert spaces of functions, each realization being similar to a choice of coordinates on a manifold. The resulting formalism is a tensor algebra of $\mathbf{S}$ that deals with the ordinary and generalized functions on an equal footing. Tensor equations in the formalism describe unitary equivalent families of functional equations on various spaces of functions. The reader is referred to Ref.[2] for mathematical considerations 
and some applications.

The sketched framework for dealing with improper states also provides a fresh look at a number of fundamental problems of quantum theory. In particular, it yields an elegant mathematical formalism for "deriving" classical space (i.e., the 3-dimensional space of everyday experience) from the space of states of a particle Ref.[3]. The starting point of the formalism is the one-to-one correspondence between points a in the classical 3-space $N_{3}$ and state functions $\delta_{\mathbf{a}}^{3}$ of a non-relativistic particle found at a. The idea is to use this correspondence to "build" $N_{3}$ from delta functions in the space of state functions. To obtain a framework independent of the classical space one drops the assumption that the state functions are defined on $N_{3}$ and introduces a Hilbert space $H$ of functions on a fixed open set $D$ in $\mathbb{R}^{3}$. If $H$ is a space of continuous functions then the dual space $H^{*}$ of continuous linear functionals on $H$ will in general contain delta functions. The key observation now is that by varying the Hilbert space $H$ (by changing the metric and choosing different Hilbert subspaces while keeping $D$ fixed) one can transform the image $M_{3}$ of the map $\omega: D \longrightarrow H^{*}$ defined by $\omega(\mathbf{a})=\delta_{\mathbf{a}}^{3}$ into a 3-manifold of an arbitrary induced topology, differentiable structure and local Riemannian metric Ref.[4]. The manifold $M_{3}$ is built of functions in a Hilbert space, not points in the domain of the functions. The variables of functions lose their physical meaning and become abstract bookkeeping indices needed to identify elements of the Hilbert space. In such a way the manifold $M_{3}$ becomes a "self sufficient" representation of the manifold $N_{3}$ without the assumption of a preexisting classical space. The manifold $N_{3}$ is now a "part" of the Hilbert space $H^{*}$, rather than the Hilbert space being built on top of the classical space. The abstract Hilbert space $\mathbf{S}$ becomes the main object of the theory, capable of representing all other objects, while the Hilbert space $H^{*}$ is the image of a particular coordinate chart on $\mathbf{S}$.

The goal of this paper is to demonstrate that the mathematical framework of Refs.[2]-[7] is also ideally suited for addressing the issues of the unification of quantum mechanics and relativity theory. First of all, it allows one to build the Minkowski space-time as a part of a Hilbert space without assuming any preexisting space-time. The Hilbert space used in the construction is a space of functions of four variables that contains the usual space of states of a nonrelativistic quantum particle as a subspace, in a certain limit. Second, for a class of functions that are "well localized" in the time variable and an appropriate evolution law the framework reproduces the standard Schrödinger mechanics. Most importantly, it provides a new covariant mathematical formalism for relativistic quantum mechanics of a single particle. In the formalism the space of physical states is an abstract Hilbert space, while spaces of state functions of a particular inertial observer are specific functional realizations of the abstract Hilbert space. In such a way the formalism is an extension of the theory of relativity. Furthermore, the framework is compatible with the Stueckelberg relativistic quantum theory and accommodates a recently observed quantum interference in time Refs.[8],[9]. At the same time, it gives an account of why the interference in time is suppressed in the non-relativistic domain and why the space and time variables play an asymmetric role in quantum mechanics. The formalism seems to be powerful enough to shed new light on other problems of relativistic quantum theory as well. Overall, the results give one hope 
that the tension between quantum theory and relativity may be resolved without denying the existence of space and time or abandoning the geometric language that proved to be so fruitful in the classical theory.

\section{THE THEOREMS}

The first step in creating a unifying framework for special relativity and quantum mechanics is to "build" the classical space from states of a particle. This must be done in a self-contained way, so that no reference to the classical space would be needed. Furthermore, one must be able to replicate the classical space in all details, including topology, differentiable structure and Riemannian metric. Following the introduction, let's begin with a Hilbert space $H_{D}$ of functions defined on an open set $D$ in $\mathbb{R}^{3}$. Define the map $\omega: D \longrightarrow H_{D}^{*}$ via $\omega(\mathbf{a})=\delta_{\mathbf{a}}^{3}$. Here the dual space $H_{D}^{*}$ is assumed to contain the functionals $\delta_{\mathbf{a}}^{3}$ for all a in $D$. The classical space $N_{3}$ can be now identified with the submanifold $M_{3}$ of $H_{D}^{*}$ formed by all delta functions in $H_{D}^{*}$ in accordance with the following result.

Theorem 1. Given an arbitrary real 3-dimensional manifold $N_{3}$ there exists a Hilbert space $H_{D}$ of continuous functions on $D$, such that the set $M_{3}$ of all delta functions in the dual space $H_{D}^{*}$ is an embedded submanifold of $H_{D}^{*}$ diffeomorphic to $N_{3}$. Moreover, if $N_{3}$ is a real analytic Riemannian manifold the above embedding of $N_{3}$ into $H_{D}^{*}$ can be assured to be locally isometric. A similar result holds true in any dimension $n$ of the manifold as well.

Proof of a particular case. The theorem claims that the whole of manifold theory in $n$-dimensions is "contained" in the theory of Hilbert spaces on an open set in $\mathbb{R}^{n}$. This general result is a version of the famous Gelfand-Kolmogorov theorem Ref.[10]. A special case of $N_{3}$ being the Euclidean space will be the subject of theorem 2. A generalization to the case of Minkowski space is discussed in theorem 4. Although these two cases are sufficient for the paper, it is enlightening to see what is involved when the topology of the classical space is non-trivial. Because the proof of a general case is rather long Ref.[4], a simple case of a one dimensional classical space modeled by a circle $S^{1}$ will be considered. In this case a Hilbert space that consists of continuous functions on $\mathbb{R}$, for which the subspace of all delta functions in the dual Hilbert space is homeomorphic to $S^{1}$ will be constructed.

Assume first that $H$ is a Hilbert space of functions on $\mathbb{R}$ that contains all smooth functions of compact support and such that the dual space $H^{*}$ contains delta functions $\delta_{a}$ for all $a \in \mathbb{R}$. In this case the map $\omega: \mathbb{R} \longrightarrow H^{*}, \omega(a)=\delta_{a}$ is one-to-one. Indeed, whenever $a \neq b$, there is a function in $H$ that takes different values at $a$ and $b$, which means that $\delta_{a}$ is not equal to $\delta_{b}$. So provided $\omega$ is continuous and the inverse map from the image $M_{1}=\omega(\mathbb{R})$ onto $\mathbb{R}$ is also continuous, the subspace $M_{1} \subset H^{*}$ is homeomorphic to $\mathbb{R}$. Accordingly, the topology of $M_{1}$ is trivial.

It follows that a non-trivial topology of $M_{1}$ depends on $\omega$ being not one-to-one. For instance, to make $M_{1}$ into a circle, delta functions $\delta_{a}, \delta_{a+2 \pi}, a \in \mathbb{R}$ must be identical. That means that all functions in the Hilbert space must take equal values at the points that are $2 \pi$ units apart. In other words, the functions must be $2 \pi$-periodic. So we need a Hilbert space of continuous $2 \pi$-periodic functions. Consider then the Hilbert space $H_{\mathbb{R}}$ of $2 \pi$-periodic functions 
on $\mathbb{R}$ with the inner product $(f, g)=\int_{-\pi}^{\pi}\left(f(x) \bar{g}(x)+f^{\prime}(x) \overline{g^{\prime}}(x)\right) d x$. This is a well known Sobolev space which is continuously embedded into the Banach space $C^{0}(\mathbb{R}, 2 \pi)$ of continuous $2 \pi$-periodic functions on $\mathbb{R}$ with the usual sup-norm: $\|f\|_{C^{0}}=\sup _{x \in[-\pi, \pi]}|f(x)|$. It follows that the dual space $H_{\mathbb{R}}^{*}$ contains delta functions $\delta_{a}$ for all $a \in \mathbb{R}$. Let's check that the subspace $M_{1} \subset H_{R}^{*}$ of delta functions in $H_{\mathbb{R}}^{*}$ is homeomorphic to the circle $S^{1}$. The norm of a function $f$ in $H_{\mathbb{R}}$ can be expressed in terms of its Fourier coefficients $\widehat{f}(k)$ as follows:

$$
\|f\|_{H_{\mathbb{R}}}^{2}=\sum_{k=-\infty}^{\infty}\left(1+k^{2}\right)|\widehat{f}(k)|^{2} .
$$

The norm of a functional $F \in H_{\mathbb{R}}^{*}$ in terms of its Fourier coefficients $\widehat{F}(k)$ is given by

$$
\|F\|_{H_{\mathbb{R}}^{*}}^{2}=\sum_{k=-\infty}^{\infty} \frac{|\widehat{F}(k)|^{2}}{1+k^{2}} .
$$

Since Fourier coefficients of $\delta_{a}(x)$ are $\frac{1}{2 \pi} e^{-i k a}$, the equation (2) yields

$$
\left\|\delta_{a_{n}}-\delta_{a}\right\|_{H_{\mathbb{R}}^{*}}^{2}=\frac{1}{\pi^{2}} \sum_{k=-\infty}^{\infty} \frac{\sin ^{2} \frac{k\left(a_{n}-a\right)}{2}}{1+k^{2}} .
$$

Recall that $S^{1}$ is the quotient space $S^{1}=\mathbb{R} / \sim$, where $a \sim b$ if $a \equiv b \bmod 2 \pi$. Let $q: \mathbb{R} \longrightarrow S^{1}$ be the quotient map and let $\widetilde{\omega}: S^{1} \longrightarrow M_{1}$ be defined by $\omega=\widetilde{\omega} \circ q$. To prove that the subspace $M_{1} \subset H_{\mathbb{R}}^{*}$ is a circle $S^{1}$ one must show that $\widetilde{\omega}$ is a homeomorphism. It is sufficient to verify that the map $\omega$ is one-to-one on $(-\pi, \pi)$, continuous on $[-\pi, \pi]$ and that if $a_{n}, a \in[-\pi, \pi]$ and $\left\|\delta_{a_{n}}-\delta_{a}\right\|_{H_{\mathbb{R}}^{*}}$ tends to zero, then $a_{n}-a$ tends to zero, or $2 \pi$. Now, $\omega$ is one-to-one on $(-\pi, \pi)$ because for all $a, b \in(-\pi, \pi)$ there exists a function $f$ in $H_{\mathbb{R}}$ such that $f(a) \neq f(b)$. Also, for $a_{n}, a \in[-\pi, \pi]$ and $a_{n}-a \longrightarrow 0$, the uniform convergence of the series in (3) gives $\left\|\delta_{a_{n}}-\delta_{a}\right\|_{H_{\mathbb{R}}^{*}} \longrightarrow 0$, which means that $\omega$ is continuous. Conversely, if $a_{n}, a \in[-\pi, \pi]$ and $\left\|\delta_{a_{n}}-\delta_{a}\right\|_{H_{\mathbb{R}}^{*}} \longrightarrow 0$, then each term of the series in (3) must converge to zero, so that $a_{n}-a$ converges to zero or $2 \pi$. It follows that $M_{1}$ is homeomorphic to $S^{1}$.

Note that the origin of a non-trivial topology on $M_{1}$ is the periodicity condition imposed on the functions, hence on the space $H_{\mathbb{R}}$, and has nothing to do with the domain $\mathbb{R}$ of the functions. Without this condition the space $M_{1}$ would be topologically trivial. In other words, a particular topology on $M_{1}$ is mathematically derived rather than being presupposed. This is possible because the space $M_{1}$ is "made of" functions rather than points in the domain of the functions. Note also that the differentiable structure on $M_{1}$ can be derived in a similar fashion Ref.[4]. If desirable, functions $f \in H_{\mathbb{R}}$ can be identified with functions $\tilde{f}$ on $S^{1}$ defined by $f=\tilde{f} \circ q$. Alternatively, since the quotient map $q$ restricted to an interval of length less than $2 \pi$ is one-to-one, functions $f \in H_{\mathbb{R}}$ restricted to such intervals can be thought of as functions in local coordinates on $S^{1}$.

Two spaces of a particular interest in the paper are the Euclidean 3-space and the Minkowski space. The following theorem yields a Hilbert space $\mathbf{H}$ that is "approximately equal" to the usual quantum-mechanical space $L_{2}\left(\mathbb{R}^{3}\right)$ of square-integrable functions on $\mathbb{R}^{3}$ and such that the space $\mathbb{R}^{3}$ with the Euclidean metric can be identified with (i.e., is isometric to) the submanifold $M_{3} \subset \mathbf{H}$. The dual space $\mathbf{H}^{*}$ will be shown to be a Hilbert space of smooth functions on 
$\mathbb{R}^{3}$. Note that because spaces of generalized functions like $\mathbf{H}$ in the theorem will be more important in the paper than their duals, the asterisk will be used to indicate the dual space of smooth functions rather than spaces of generalized functions, as before.

Theorem 2. The Hilbert space $\mathbf{H}$ obtained by completing the space $L_{2}\left(\mathbb{R}^{3}\right)$ in the metric defined by the inner product

$$
(\varphi, \psi)_{\mathbf{H}}=\left(\frac{L}{\sqrt{2 \pi}}\right)^{3} \int e^{-\frac{L^{2}}{2}(\mathbf{x}-\mathbf{y})^{2}} \varphi(\mathbf{x}) \bar{\psi}(\mathbf{y}) d^{3} \mathbf{x} d^{3} \mathbf{y}
$$

with a positive constant $L$ contains delta functions and their derivatives. The map $\omega: a \longrightarrow \delta_{\mathbf{a}}^{3}$ is an isometric embedding of the space $\mathbb{R}^{3}$ with the Euclidean metric into $\mathbf{H}$. Finally, for a sufficiently large $L$ the $\mathbf{H}$ and $L_{2}$-norms of any given function $f \in L_{2}\left(\mathbb{R}^{3}\right)$ are arbitrarily close to each other.

Sketch of a proof. The operator $\rho: L_{2}\left(\mathbb{R}^{3}\right) \longrightarrow L_{2}\left(\mathbb{R}^{3}\right)$ defined by $(\rho f)(\mathbf{x})=\left(\frac{L}{\sqrt{2 \pi}}\right)^{3 / 2} \int e^{-L^{2}(\mathbf{x}-\mathbf{y})^{2}} f(\mathbf{y}) d \mathbf{y}$ is invertible. The image of this map is a separable Hilbert space $\mathbf{H}^{*}$ of analytic functions with the inner product $(F, G)_{\mathbf{H}^{*}}=\left(\rho^{-1} F, \rho^{-1} G\right)_{L_{2}}$. (The isomorphism $\rho$ is a particular case of the Segal-Bargmann transform Refs.[11],[12]). The space $\mathbf{H}^{*}$ is continuously embedded into $L_{2}\left(\mathbb{R}^{3}\right)$ as a dense subset so that the dual space $\mathbf{H}$ is the completion of $L_{2}\left(\mathbb{R}^{3}\right)$ in the metric $(\varphi, \psi)_{\mathbf{H}}=\left(\rho^{*} \varphi, \rho^{*} \psi\right)_{L_{2}}$. Here $\rho^{*}: \mathbf{H} \longrightarrow L_{2}\left(\mathbb{R}^{3}\right)$ is the adjoint operator and the dual space $L_{2}^{*}\left(\mathbb{R}^{3}\right)$ is identified with $L_{2}\left(\mathbb{R}^{3}\right)$ in the obvious way. From the definition of $\rho$ one concludes that the inner product on $\mathbf{H}$ is given by (4). Note that the integral sign is used as a symbol of action of the inner product form on $\mathbf{H} \times \mathbf{H}$. It can be shown that $\mathbf{H}^{*}$ is continuously embedded into any of the Banach spaces $C_{0}^{k}\left(\mathbb{R}^{3}\right)$ of $k$-times continuously differentiable, decreasing at infinity functions with the usual supremum norm. Therefore $\mathbf{H}$ contains delta functions and their derivatives. The fact that $\omega$ is an isometric embedding of the space $\mathbb{R}^{3}$ with the Euclidean metric into $\mathbf{H}$ will be verified in a more general setting in theorem 4. Finally, the kernels $\left(\frac{L}{\sqrt{2 \pi}}\right)^{3} e^{-\frac{L^{2}}{2}(\mathbf{x}-\mathbf{y})^{2}}$ form an approximation of the identity. That means that for any $\psi \in L_{2}\left(\mathbb{R}^{3}\right)$ the expression $\left(\frac{L}{\sqrt{2 \pi}}\right)^{3} \int e^{-\frac{L^{2}}{2}(\mathbf{x}-\mathbf{y})^{2}} \psi(\mathbf{y}) \mathbf{d y}$ tends to $\psi(\mathbf{x})$ in the $L_{2}$-norm as $L$ increases. It follows that $\|\psi\|_{\mathbf{H}}$ tends to $\|\psi\|_{L_{2}}$ as $L$ increases. The details can be found in Ref.[2].

The proved relationship of $\mathbf{H}$ with $L_{2}\left(\mathbb{R}^{3}\right)$ will be symbolically referred to by writing $\mathbf{H} \approx L_{2}\left(\mathbb{R}^{3}\right)$. In the following the Planck system of units will be used. It is not an indication of the relationship to the Planck scale, but a choice of units. In particular, the speed of light factor in relativistic formulas will be absent. To further simplify the formulas, the constant $L$ in the metric (4) will be set to 1 . The resulting kernel $\frac{1}{(2 \pi)^{3 / 2}} e^{-\frac{1}{2}(\mathbf{x}-\mathbf{y})^{2}}$ of the metric falls off to almost zero within the first few Planck units $\left(\approx 10^{-35} \mathrm{~m}\right)$ of the Euclidean distance $\|\mathbf{x}-\mathbf{y}\|_{\mathbb{R}^{3}}$. Accordingly, the norms of the typical square-integrable functions in the space $\mathbf{H}$ defined by (4) with $L=1$ will be extremely close to their norms in the space $L_{2}\left(\mathbb{R}^{3}\right)$. It follows that the expected values, probabilities of transitions and other measured quantities remain practically the same in the new metric, ensuring consistency with experiment. Of course, a much smaller value of $L$ may be sufficient for the consistency. So the value of $L$ will be kept open within the consistency constraint. Let us also agree to set the constant factor in front of the metric to 1 . This does not change the relative probabilities of events expressed in terms of the H-metric, and makes the delta functions unit-normalized. 
The classical space $\mathbb{R}^{3}$ with the Euclidean metric is now a submanifold of the Hilbert space $\mathbf{H} \approx L_{2}\left(\mathbb{R}^{3}\right)$ formed by point-supported state functions $\delta_{\mathbf{a}}^{3}$. To obtain a relativistic framework one needs to consider spaces of functions of four variables $x=(\mathbf{x}, t)$. The following two theorems provide a generalization of the theorem 2 to this case.

Theorem 3. Let $\widetilde{H}$ be the Hilbert space of functions $f$ of four variables $x=(\mathbf{x}, t)$ that is the completion of the space $L_{2}\left(\mathbb{R}^{4}\right)$ in the metric given by the kernel $e^{-\frac{1}{2}(x-y)^{2}}$. Let $\widetilde{H}_{\mathrm{ev}}$ be the subspace of all functions in $\widetilde{H}$ that are even in the time variable $t$. Likewise, let $\widetilde{H}_{\text {odd }}$ be the subspace of all functions that are odd in $t$. Then $\widetilde{H}$ is the orthogonal sum of $\widetilde{H}_{\mathrm{ev}}$ and $\widetilde{H}_{\mathrm{odd}}$. Furthermore, consider the set $H$ of all functions $f(\mathbf{x}, t)=e^{-t^{2}} \varphi(\mathbf{x}, t)$ with $\varphi \in \widetilde{H}$. Consider the Hermitian form $(f, g)_{H_{\eta}}$ on $H$ given by

$$
(f, g)_{H_{\eta}}=\int e^{-\frac{1}{2}(\mathbf{x}-\mathbf{y})^{2}+\frac{1}{2}(t-s)^{2}} f(\mathbf{x}, t) \bar{g}(\mathbf{y}, s) d^{3} \mathbf{x} d t d^{3} \mathbf{y} d s
$$

and let $(f, f)_{H_{\eta}} \equiv\|f\|_{H_{\eta}}^{2}$ be the corresponding quadratic form, or the squared $H_{\eta}$-norm. Then $H$ is exactly the set of functions whose even and odd components have a finite $H_{\eta}$-norm. Moreover, $H$ furnished with the inner product $(f, g)_{H_{+}}=(\varphi, \psi)_{\widetilde{H}}$ where $f(\mathbf{x}, t)=e^{-t^{2}} \varphi(\mathbf{x}, t), g(\mathbf{x}, t)=e^{-t^{2}} \psi(\mathbf{x}, t)$ is a Hilbert space. The Hermitian form (5) defines an indefinite, non-degenerate inner product on $H$, such that $\|f\|_{H_{\eta}}^{2}>0$ for all even functions $f \neq 0$ and $\|f\|_{H_{\eta}}^{2}<0$ for all odd functions $f \neq 0$ in $H$. Finally, $H$ contains the delta functions $\delta_{a}^{4}(x)=\delta^{4}(x-a)$ and their derivatives.

Proof. Assume first that $f \in \tilde{H}$ is continuous on $\mathbb{R}^{4}$. Note that $f$ can be uniquely written as a sum of even and odd functions of $t$ via $f(\mathbf{x}, t)=\frac{1}{2}(f(\mathbf{x}, t)+f(\mathbf{x},-t))+\frac{1}{2}(f(\mathbf{x}, t)-f(\mathbf{x},-t))$. Also, if $f(\mathbf{x}, t)$ is even and $g(\mathbf{y}, s)$ is odd in the time variable, then $(f, g)_{\tilde{H}}$ vanishes. In fact, integration over the first quadrant in the st-plane gives the result that is opposite to the integral over the third quadrant, because the integrand changes sign under the transformation $(s, t) \longrightarrow(-s,-t)$. Similarly, integration with respect to the second and the fourth quadrants cancel out. Since continuous functions are dense in $\widetilde{H}$ and the property of being even/odd is preserved under taking a limit, the space $\widetilde{H}$ is the orthogonal sum $\widetilde{H}_{\text {ev }} \oplus \widetilde{H}_{\text {odd }}$ of subspaces consisting respectively of even or odd functions of $t$.

Let $f$ be a continuous function on $\mathbb{R}^{4}$ and let $f(\mathbf{x}, t)=e^{-t^{2}} \varphi(\mathbf{x}, t)$ for some function $\varphi$. Then $\|f\|_{H_{\eta}}^{2}$ is equal to

$$
\int e^{-\frac{1}{2}(\mathbf{x}-\mathbf{y})^{2}-\frac{1}{2}(t+s)^{2}} \varphi(\mathbf{x}, t) \bar{\varphi}(\mathbf{y}, s) d^{3} \mathbf{x} d t d^{3} \mathbf{y} d s
$$

If $\varphi \in \widetilde{H}_{\mathrm{ev}}$ then changing $s$ to $-s$ in the integral one obtains

$$
\int e^{-\frac{1}{2}(\mathbf{x}-\mathbf{y})^{2}-\frac{1}{2}(t-s)^{2}} \varphi(\mathbf{x}, t) \bar{\varphi}(\mathbf{y}, s) d^{3} \mathbf{x} d t d^{3} \mathbf{y} d s
$$

This is equal to $(\varphi, \varphi)_{\widetilde{H}}$ so that $\|f\|_{H_{\eta}}^{2}=\|\varphi\|_{\widetilde{H}}^{2}$ is positive definite whenever $f$ is even in $t$. Notice that $\|f\|_{H_{\eta}}^{2}$ is finite if and only if $\|\varphi\|_{\widetilde{H}}^{2}$ is finite. Moreover, the set $H_{\mathrm{ev}}$ of all functions $f(\mathbf{x}, t)=e^{-t^{2}} \varphi(\mathbf{x}, t)$ with $\varphi \in \widetilde{H}_{\mathrm{ev}}$ is a Hilbert space relative to the inner product $(f, g)_{H_{+}}=(\varphi, \psi)_{\tilde{H}}$, where $f(\mathbf{x}, t)=e^{-t^{2}} \varphi(\mathbf{x}, t)$ and $g(\mathbf{x}, t)=e^{-t^{2}} \psi(\mathbf{x}, t)$.

Suppose now that $\varphi \in \widetilde{H}_{\text {odd }}$. By the same change of variables in (6) one obtains an expression that is the opposite of (7). So the quadratic form $\|f\|_{H_{\eta}}^{2}$ is negative definite on the odd functions of $t$. By the same argument as before 
the space $H_{\text {odd }}$ of all functions $f(\mathbf{x}, t)=e^{-t^{2}} \varphi(\mathbf{x}, t), \varphi \in \widetilde{H}_{\text {odd }}$ is a Hilbert space relative to the inner product $(f, g)_{H_{+}}=(\varphi, \psi)_{\widetilde{H}}$ with $f(\mathbf{x}, t)=e^{-t^{2}} \varphi(\mathbf{x}, t), g(\mathbf{x}, t)=e^{-t^{2}} \psi(\mathbf{x}, t)$.

Consider now the space $H$ of all functions $f(\mathbf{x}, t)=e^{-t^{2}} \varphi(\mathbf{x}, t), \varphi \in \widetilde{H}$ with the positive definite inner product $(f, g)_{H_{+}}=(\varphi, \psi)_{\widetilde{H}}$ where $f(\mathbf{x}, t)=e^{-t^{2}} \varphi(\mathbf{x}, t), g(\mathbf{x}, t)=e^{-t^{2}} \psi(\mathbf{x}, t)$ and $\varphi, \psi \in \widetilde{H}$. The space $H$ with this inner product is Hilbert and $H_{\mathrm{ev}}, H_{\mathrm{odd}}$ are orthogonal subspaces of $H$ so that $H=H_{\mathrm{ev}} \oplus H_{\mathrm{odd}}$. Notice that the subspaces $H_{\mathrm{ev}}, H_{\mathrm{odd}}$ are also orthogonal in the indefinite metric $(f, g)_{H_{\eta}}$ on $H$. Clearly, the space $H$ is the space of functions $f$ whose even and odd components $f_{\text {ev }}, f_{\text {odd }}$ have a finite $H$-norm. Note that in terms of the Hilbert metric, the indefinite norm of a function $f \in H$ is given by $\|f\|_{H_{\eta}}^{2}=\left\|f_{\text {ev }}\right\|_{H_{+}}^{2}-\left\|f_{\text {odd }}\right\|_{H_{+}}^{2}$. In particular, if a sequence of functions converges in the $H_{+}$-metric it will also converge in the $H_{\eta}$-metric. Note also that the positive-definite inner product on $H$ is given more directly by $(f, g)_{H_{+}}=\int e^{-\frac{1}{2}(\mathbf{x}-\mathbf{y})^{2}+\frac{1}{2}(t+s)^{2}} f(\mathbf{x}, t) \bar{g}(\mathbf{y}, s) d \mathbf{x} d t d \mathbf{y} d s$.

The form $(f, g)_{H_{\eta}}$ is non-degenerate. In fact, if $f \neq 0$, then either the even $f_{\text {ev }}$ or the odd $f_{\text {odd }}$ components of $f$ do not vanish. If, say, $f_{\mathrm{ev}} \neq 0$, then $\left(f, f_{\mathrm{ev}}\right)_{H_{\eta}}=\left(f_{\mathrm{ev}}, f_{\mathrm{ev}}\right)_{H_{\eta}} \neq 0$. Similarly, if $f_{\mathrm{odd}} \neq 0$, then $\left(f, f_{\mathrm{odd}}\right)_{H_{\eta}}=$ $\left(f_{\text {odd }}, f_{\text {odd }}\right)_{H_{\eta}} \neq 0$.

The space $\widetilde{H}$ is known to contain delta functions and their derivatives (see theorem 2 and Ref.[2]). Because the elements of $H$ have the form $f(\mathbf{x}, t)=e^{-t^{2}} \varphi(\mathbf{x}, t)$ with $\varphi \in \widetilde{H}$, one concludes that these functionals are also in $H$.

The space $H$ in the theorem has a structure of what is called a Krein space. Note that $H$ is a subspace in the space $\widetilde{H}$. However, this subspace is not closed. In fact, the set of continuous functions of bounded support belongs to $H$ and is dense in $\widetilde{H}$. In order to make $H$ a complete space, the $H_{+}$-metric was used in the theorem. Still, in some problems it will be useful to consider $H$ as a (non-closed) subspace of $\widetilde{H}$ with the $\widetilde{H}$-metric.

The next step is to prove that the space $H$ is an appropriate arena for classical relativistic mechanics. For this, consider the map $\omega: a \longrightarrow \delta_{a}^{4}$ from the Minkowski space $N$ into the Hilbert space $H$ constructed in theorem 3 and let $M_{4}=\omega\left(R^{4}\right) \subset H$ be the image of $\omega$. The following theorem characterizes the map and its image.

Theorem 4. The map $\omega$ is an embedding that identifies the Minkowski space $N$ with the submanifold $M_{4}$ of $H$ of all delta functions $\delta_{\mathbf{a}}^{4}, \mathbf{a} \in N$. Under the embedding the indefinite metric on $H$ yields the Minkowski metric on $M_{4}$, while the $\widetilde{H}$-metric yields the ordinary Euclidean metric on $M_{4}$.

Proof. The map $\omega$ is injective because $H^{*}$ contains sufficiently many functions, so that whenever $a \neq b$, there exists a function $F \in H^{*}$ such that $F(a) \neq F(b)$. The derivative map $d \omega$ is non-singular because the derivatives $\frac{\partial \delta_{a}^{4}(x)}{\partial x^{\alpha}}$ of delta functions with respect to coordinates $x^{\alpha}$ on $N$ are linearly independent functionals in $H$. The distance between two delta functions $\delta_{a}^{4}, \delta_{b}^{4}, a=\left(\mathbf{a}, a_{0}\right), b=\left(\mathbf{b}, b_{0}\right)$ in the Hilbert space $H$ is given by

$$
\left\|\delta_{a}-\delta_{b}\right\|_{H_{+}}^{2}=e^{2 a_{0}^{2}}+e^{2 b_{0}^{2}}-2 e^{-\frac{1}{2}(\mathbf{a}-\mathbf{b})^{2}+\frac{1}{2}\left(a_{0}+b_{0}\right)^{2}}=\left(e^{a_{0}^{2}}-e^{b_{0}^{2}}\right)^{2}+2 e^{a_{0}^{2}+b_{0}^{2}}\left(1-e^{-\frac{1}{2}(a-b)^{2}}\right)
$$


The same distance in the $\widetilde{H}$-metric is given by

$$
\left\|\delta_{a}-\delta_{b}\right\|_{\widetilde{H}}^{2}=2-2 e^{-\frac{1}{2}(a-b)^{2}}
$$

From (8) and (9) one concludes that for the usual $\mathbb{R}^{4}$-topology on $N$ and either $H_{+}$or $\widetilde{H}$-topologies on $H$ (and the induced topology on $M_{4}$ ) the map $\omega$ and its inverse $\omega^{-1}: M_{4} \longrightarrow N$ are continuous. To derive an expression for the induced metric on $M_{4}$ in coordinates $x^{\alpha}$ on $N$ consider the inner products of the velocity vectors $\left.\frac{d f_{\tau}(x)}{d \tau}\right|_{\tau=0}$ of the curves $f_{\tau}(x)=\delta_{a+u \tau}^{4}$ passing through $\delta_{a}$ in the direction specified by vector $u$. In particular,

$$
\left(\frac{d f_{\tau}}{d \tau}, \frac{d f_{\tau}}{d \tau}\right)_{H_{\eta}}=\int k_{\eta}(x, y) \frac{\partial \delta_{a}^{4}(x)}{\partial x^{\alpha}} \frac{\partial \delta_{a}^{4}(y)}{\partial y^{\beta}} u^{\alpha} u^{\beta} d^{4} x d^{4} y
$$

where $k_{\eta}(x, y)=e^{-\frac{1}{2}(\mathbf{x}-\mathbf{y})^{2}+\frac{1}{2}(t-s)^{2}}$ is the kernel of the indefinite metric on $H$. This gives

$$
\left(\frac{d f_{\tau}}{d \tau}, \frac{d f_{\tau}}{d \tau}\right)_{H_{\eta}}=\left.\frac{\partial^{2} k_{\eta}(x, y)}{\partial x^{\alpha} \partial y^{\beta}}\right|_{x=y=a} u^{\alpha} u^{\beta}=\eta_{\alpha \beta} u^{\alpha} u^{\beta}
$$

where $\eta$ is the Minkowski tensor of signature $(-,+,+,+)$. It follows that the induced metric is the Minkowski metric while the subspace topology is the topology of the Euclidean space $\mathbb{R}^{4}$. By replacing the kernel $k_{\eta}(x, y)$ with the kernel $k_{\widetilde{H}}(x, y)=e^{-\frac{1}{2}(x-y)^{2}}$ of the $\widetilde{H}$-metric, one obtains the Euclidean metric on $M_{4}$.

So the set $M_{4}$ with the induced topology of the space $\mathbb{R}^{4}$ and Minkowski metric is identical (isometric) to the usual Minkowski space. For instance, if the world line of a massive particle in Minkowski space is given by $x^{\alpha}=a^{\alpha}(\tau)$, where $\tau$ is a proper time parameter, then the velocity $\frac{d \varphi_{\tau}}{d \tau}$ of the path $\varphi_{\tau}=\delta_{a(\tau)}^{4}$ in $M_{4} \subset H$ is equal to the 4 -velocity $\frac{d a^{\alpha}}{d \tau}$. Likewise, the distance along the curve $\varphi_{\tau}$ in the indefinite metric on $H$ is equal to the ordinary 4-distance traveled by the particle in Minkowski space $N$ :

$$
\int\left\|\frac{d \varphi_{\tau}}{d \tau}\right\|_{H_{\eta}} d \tau=\int\left\|\frac{d a}{d \tau}\right\|_{\eta} d \tau
$$

Note that the indefinite norm of a delta function $\delta_{a}$ is one. In particular, the Minkowski space $N$ is a submanifold of the infinite-dimensional hyperboloid $\|f\|_{H_{\eta}}^{2}=1$ in the space $H$. Because the $\widetilde{H}$-norm of $\delta_{a}$ is also one, $N$ is a submanifold of the unit sphere $\|f\|_{\widetilde{H}}^{2}=1$ in the space $\widetilde{H}$ as well. The following theorem explains the way in which $N$ is embedded into $H$ and $\widetilde{H}$.

Theorem 5. The set $M_{4}$ is a complete set in the Hilbert spaces $H$ and $\widetilde{H}$. Accordingly, linear combinations of elements of $M_{4}$ span these spaces. The elements of any finite subset of $M_{4}$ are linearly independent. No two elements of $M_{4}$ are orthogonal in the indefinite metric or the Hilbert metric on $H$, or the $\widetilde{H}$-metric. However, the inner product $\left(\delta_{a}^{4}, \delta_{b}^{4}\right)_{\widetilde{H}}$ with $a=\left(\mathbf{a}, a_{0}\right)$ and $b=\left(\mathbf{b}, b_{0}\right)$ tends to zero when the Euclidean norm $\|a-b\|_{R^{4}}$ tends to infinity. Similarly, the indefinite inner product $\left(\delta_{a}^{4}, \delta_{b}^{4}\right)_{H_{\eta}}$ tends to zero when the Minkowski norm $\|a-b\|_{N}^{2}=(\mathbf{a}-\mathbf{b})^{2}-\left(a_{0}-b_{0}\right)^{2}$ tends to infinity. 
Proof. Assume that $\left(f, \delta_{a}\right)_{H_{+}}=0$ for all $a \in N$. Then $\widehat{G}_{H_{+}} f=0$, where $\widehat{G}_{H_{+}}: H \longrightarrow H^{*}$ is the operator that defines the metric on $H$. But this operator is an isomorphism, so that $f=0$. The same is true in the $\widetilde{H}$-metric. It follows that $M_{4}$ is a complete set in $H$ and $\widetilde{H}$. Other statements follow directly from the definitions.

Because $M_{4}$ is a complete set in $\widetilde{H}$ it is not contained in any finite dimensional subspace of $\widetilde{H}$. For instance, under the embedding $\omega: a \longrightarrow \delta_{a}^{4}$ a straight line $x=x_{0}+a \tau$ in Minkowski space becomes a curve on the sphere $S^{\widetilde{H}}$. This curve contains infinitely many linearly independent vectors of $\widetilde{H}$. In passing along the sphere the curve "sticks out" of any finite dimensional subspace of $\widetilde{H}$ winding through dimensions of $\widetilde{H}$.

Note that the manifold $M_{4}$ is not a linear subspace in $H$ : the map $\omega$ is not linear. At the same time one can introduce a linear structure on $M_{4}$ by defining the operations of addition $\oplus$ and multiplication by a scalar $\lambda \odot$ via $\omega(a) \oplus \omega(b)=\omega(a+b)$ and $\lambda \odot \omega(a)=\omega(\lambda a)$. Moreover, because $\omega$ is a homeomorphism onto $M_{4}$, these operations are continuous in the topology of $M_{4} \subset H$. The same applies to $M_{4}$ as a submanifold of $\widetilde{H}$.

Classical space-time $N$ is now embedded into the Hilbert space $H$ of functions of four variables $\mathbf{x}, t$. The immediate question is whether the theory of relativity on $N$ can be also realized as a theory on $H$. The definition of $H$ in theorem 3 is tied to a particular reference frame. The Poincaré invariance of the construction can be then questioned. The following theorem defines an essentially unique relativistic invariant extension of the mathematical structure of special relativity to the abstract Hilbert space. The word "isomorphism" in the statement of the theorem refers to an isomorphism of Hilbert spaces that also preserves the indefinite metric. Likewise, the "isometric embedding" is an embedding that preserves the indefinite metric. Note also that the Minkowski space, and the Hilbert space are purely geometric constructions that do not require any specific realization. For instance, no particular reference frame (coordinate system) is needed to define the Minkowski space. Similarly, no particular space of functions is needed to define the abstract Hilbert space. Accordingly, symbol $N$ in the theorem will be used for the abstract-geometric Minkowski space, while the symbol $\mathbb{R}^{1,3}$ will refer to a particular coordinate realization of $N$. Likewise, symbol $\mathbf{S}$ will be used for the abstract Hilbert space, while $H$ and $H^{\prime}$ will be used for specific functional realizations of $\mathbf{S}$.

Theorem 6. Let $\Gamma: \mathbf{S} \longrightarrow H$ be an isomorphism of the abstract Hilbert space $\mathbf{S}$ with an additional indefinite metric onto the space $H$ of functions defined in theorem 3. Let $\gamma: N \longrightarrow \mathbb{R}^{1,3}$ be a global coordinate chart from the Minkowski space-time onto the coordinate space of observer in an inertial reference frame $K$. Let $\Pi$ be a Poincaré transformation that relates coordinates of the frames $K$ and $K^{\prime}$. Then there exists a unique isometric embedding $\Omega$ and a unique isomorphism $\delta_{\Pi}: H \longrightarrow H^{\prime}$ such that the diagram

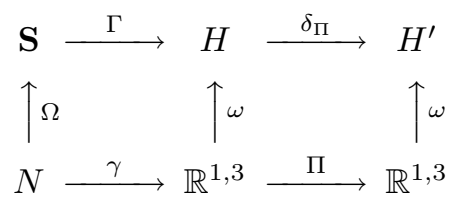

commutes. It follows that the embedding $\omega$ preserves the structure of special relativity and extends it in a unique way 
to the abstract Hilbert space $\mathbf{S}$.

Proof. From the diagram one can see that $\Omega=\Gamma^{-1} \omega \gamma$. Since by theorem 4 the map $\omega$ is an isometric embedding and $\gamma, \Gamma$ preserve the indefinite metric, the map $\Omega$ is an isometric embedding as well. Provided $\delta_{\Pi}$ in the statement exists, its restriction to the image $\omega\left(\mathbb{R}^{1,3}\right)=M_{4}$ of $\omega$ is given by $\widetilde{\delta}_{\Pi}=\omega \Pi \omega^{-1}$. The latter transformation maps $\delta_{\mathbf{a}}^{4}$ to $\delta_{\text {Пa }}^{4}$, so its kernel must be $\delta^{4}\left(\Pi^{-1} x^{\prime}-x\right)$. Indeed, $\int \delta^{4}\left(\Pi^{-1} x^{\prime}-x\right) \delta^{4}(x-a) d^{4} x=\delta^{4}\left(\Pi^{-1} x^{\prime}-a\right)=\delta^{4}\left(x^{\prime}-\Pi a\right)$. A linear bijective extension of $\widetilde{\delta}_{\Pi}$ to the space $H$ is defined by the same kernel via $\left(\delta_{\Pi} f\right)\left(x^{\prime}\right)=\int \delta^{4}\left(\Pi^{-1} x^{\prime}-x\right) f(x) d^{4} x=f\left(\Pi^{-1} x^{\prime}\right)$. Such an extension is a map onto the linear space $H^{\prime}$ of all compositions $f \circ \Pi^{-1}, f \in H$. The extension $\delta_{\Pi}$ is an isomorphism if and only if $H^{\prime}$ is furnished with the Hilbert and the indefinite metrics defined by $\left(\delta_{\Pi} f, \delta_{\Pi} g\right)_{H_{+}^{\prime}}=(f, g)_{H_{+}}$and $\left(\delta_{\Pi} f, \delta_{\Pi} g\right)_{H_{\eta}^{\prime}}=(f, g)_{H_{\eta}}$ for all $f, g \in H$. Finally, the extension $\delta_{\Pi}$ is unique because the set $M_{4}$ is complete in $H$. This proves the existence and uniqueness of the isomorphism $\delta_{\Pi}$ in the diagram and completes the proof.

Note that a function $f \in H$ represents an invariant element of $\mathbf{S}$ and transforms as a vector under $\delta_{\Pi}: f^{\prime}=\delta_{\Pi} f$. The metric operators $\widehat{G}_{H_{+}}, \widehat{G}_{H_{\eta}}: H \longrightarrow H^{*}$, where $H^{*}$ is the dual of $H$, define 2-forms in the tensor algebra of $\mathbf{S}$. Their transformation law $\widehat{G}_{H_{+}^{\prime}}=\delta_{\Pi}^{*-1} \widehat{G}_{H_{+}} \delta_{\Pi}^{-1}$ and $\widehat{G}_{H_{\eta}^{\prime}}=\delta_{\Pi}^{*-1} \widehat{G}_{H_{\eta}} \delta_{\Pi}^{-1}$, where $\delta_{\Pi}^{*}: H^{\prime *} \longrightarrow H^{*}$ is the adjoint of $\delta_{\Pi}$, ensures invariance of the inner products. Writing the law $f^{\prime}=\delta_{\Pi} f$ in the form $f^{\prime}\left(x^{\prime}\right)=\left(\delta_{\Pi} f\right)\left(x^{\prime}\right)=f\left(\Pi^{-1} x^{\prime}\right)=f(x)$, one recovers the usual law of transformation of scalar functions. In other words, the vector law of the transformation of $f$ under $\delta_{\Pi}$ is "compensated" by the corresponding change of coordinates. Note that more general functional transformations of $f$ cannot be reduced to a coordinate change and represent a new kind of symmetry that may be relevant to physics.

Let us call the realization $\Gamma: \mathbf{S} \longrightarrow H$ of $\mathbf{S}$ a $K$-representation. The diagram (13) demonstrates that under the transformation of the frame $K$ by $\gamma \longrightarrow \Pi \circ \gamma$ the $K$-representation changes in a covariant fashion to a unitary equivalent realization $\delta_{\Pi} \circ \Gamma: \mathbf{S} \longrightarrow H^{\prime}$ of $\mathbf{S}$. According to the diagram, this realization is a unique extension of the coordinate system $\Pi \circ \gamma$ of an observer in the reference frame $K^{\prime}$, or the $K^{\prime}$-representation of $\mathbf{S}$. Note that in general the spaces $H$ and $H^{\prime}$ have a different functional content. However, both spaces are realizations of the same invariant abstract Hilbert space $\mathbf{S}$ with the invariant Hilbert and indefinite metrics on it. In other words, only a functional realization of $\mathbf{S}$ changes from frame to frame, not the space $\mathbf{S}$ itself. For applications to physics it is particularly important that the inner products of elements of $\mathbf{S}$ in all realizations remain the same.

Having realized the Minkowski space $N$ as the submanifold $M_{4}$ of $H$ and the action of groups on $N$ via isomorphisms of $H$ that map $M_{4}$ onto itself, one concludes that relativity theory on Minkowski space-time is now realized as a theory on a Hilbert space. The next step in providing a unifying mathematical framework for quantum theory and special relativity is to prove that the usual apparatus of the non-relativistic quantum mechanics is also included in the formalism. The first challenge is to relate the constructed Hilbert space $H$ of functions of four variables to the usual spaces of state functions of three spatial variables with $t$ as a parameter of evolution. For this consider the family of 
subspaces $H_{\tau}$ of $H$ each consisting of all functionals $\varphi_{\tau}$ of the form $\varphi_{\tau}(\mathbf{x}, t)=\psi(\mathbf{x}, t) \delta(t-\tau)$ for some fixed $\tau \in \mathbb{R}$.

Theorem 7. Under the inclusion $i: H_{\tau} \longrightarrow H$ the indefinite inner product on $H$ yields a Hilbert metric on $H_{\tau}$ for all $\tau \in \mathbb{R}$. The inner products $H_{+}$and $\widetilde{H}$ on $H$ yield an equivalent Hilbert metric on $H_{\tau}$. Furthermore, let $\mathbf{H} \approx L_{2}\left(\mathbb{R}^{3}\right)$ be the Hilbert space defined in theorem 2. Then for all $\tau \in \mathbb{R}$ the map $I: H_{\tau} \longrightarrow \mathbf{H}$ defined by $I\left(\varphi_{\tau}(\mathbf{x}, t)\right)=\psi(\mathbf{x}, \tau)$ is an isomorphism of Hilbert spaces.

Proof. If $\varphi_{\tau}(\mathbf{x}, t)=\psi(\mathbf{x}, t) \delta(t-\tau)$ and $\chi_{\tau}(\mathbf{x}, t)=\rho(\mathbf{x}, t) \delta(t-\tau)$, then

$$
\left(\varphi_{\tau}, \chi_{\tau}\right)_{H_{\eta}}=\int e^{-\frac{1}{2}(\mathbf{x}-\mathbf{y})^{2}} \psi(\mathbf{x}, \tau) \bar{\rho}(\mathbf{y}, \tau) d \mathbf{x} d \mathbf{y}
$$

The $\widetilde{H}$-inner product of these functions yields the same expression, while the $H_{+}$-inner product is different by a factor $e^{2 \tau^{2}}$, so that the resulting metrics are all equivalent. For any given $\tau$ the space $H_{\tau}$ is a closed subspace of $\widetilde{H}$, so it is a Hilbert space. The map $I$ is obviously linear and one-to-one. Since the right hand side of (14) is the inner product of $\psi, \rho$ in $\mathbf{H}, I$ is an isomorphism of the Hilbert spaces $H_{\tau}$ and $\mathbf{H}$.

The map $I$ basically identifies each subspace $H_{\tau}$ with the usual space $L_{2}\left(\mathbb{R}^{3}\right)$ of state functions on $\mathbb{R}^{3}$ considered at time $\tau$. As discussed in the theorem, the Hilbert metric on $H$ restricted to $H_{\tau}$ yields a metric equivalent to the one given by (14). Moreover, both metrics are exactly the same in the space $H_{T}$ of the "time co-moving" representation, defined by the isomorphism $\left(\delta_{\Pi} f\right)(\mathbf{x}, t)=f(\mathbf{x}, t-\tau)$ applied to $H$. This does not change the indefinite Hilbert metric while the kernel of the Hilbert metric becomes $e^{-\frac{1}{2}(\mathbf{x}-\mathbf{y})^{2}+\frac{1}{2}(t+s-2 \tau)^{2}}$. The induced metric on $H_{\tau}$ is then given by

$$
\int e^{-\frac{1}{2}(\mathbf{x}-\mathbf{y})^{2}+\frac{1}{2}(t+s-2 \tau)^{2}} \psi(\mathbf{x}, t) \delta(t-\tau) \bar{\rho}(\mathbf{y}, \tau) \delta(s-\tau) d \mathbf{x} d t d \mathbf{y} d s=\int e^{-\frac{1}{2}(\mathbf{x}-\mathbf{y})^{2}} \psi(\mathbf{x}, \tau) \bar{\rho}(\mathbf{y}, \tau) d \mathbf{x} d \mathbf{y}
$$

which coincides with (14).

The next task is to relate the dynamics on the family of subspaces $H_{\tau}$ and the usual space $L_{2}\left(\mathbb{R}^{3}\right)$ of states of a spinless non-relativistic particle.

Theorem 8. Let $\widehat{h}=D+V(\mathbf{x}, t)$ be a Hamiltonian, such that $D$ is a differential operator in the spatial coordinates and $V$ is a function. Then the path $\varphi_{\tau}(\mathbf{x}, t)=\psi(\mathbf{x}, t) \delta(t-\tau)$ in $H$ satisfies the equation $\frac{d \varphi_{\tau}}{d \tau}=\left(-\frac{\partial}{\partial t}-i \widehat{h}\right) \varphi_{\tau}$ if and only if the function $\psi(\mathbf{x}, t)$ satisfies the Schrödinger equation $\frac{\partial \psi(\mathbf{x}, t)}{\partial t}=-i \widehat{h} \psi(\mathbf{x}, t)$. At each point of the path $\varphi_{\tau}$ the components $-i \widehat{h} \varphi_{\tau},-\frac{\partial \varphi_{\tau}}{\partial t}$ of the velocity vector $\frac{d \varphi_{\tau}}{d \tau}$ are orthogonal in the indefinite inner product, $\widetilde{H}-i n n e r$ product and the inner product on the space $H_{T}$ of the time co-moving representation.

Proof. The fact that the equation $\frac{d \varphi_{\tau}}{d \tau}=\left(-\frac{\partial}{\partial t}-i \widehat{h}\right) \varphi_{\tau}$ for the path $\varphi_{\tau}(\mathbf{x}, t)=\psi(\mathbf{x}, t) \delta(t-\tau)$ is equivalent to the Schrödinger equation $\frac{\partial \psi}{\partial t}=-i \widehat{h} \psi$ is verified directly by comparing the derivatives of $\varphi_{\tau}(\mathbf{x}, t)$ with respect to $t$ and $\tau$. Furthermore, $\frac{\partial \psi(\mathbf{x}, t)}{\partial t} \delta(t-\tau)$ is in $H_{\tau}$ (provided $\frac{\partial \psi}{\partial t} \in \mathbf{H}$ ) and so $-i \widehat{h} \varphi_{\tau}$ is in $H_{\tau}$ for all $\tau$. If $\chi \in H_{\tau}$, $\chi(\mathbf{x}, t)=\eta(\mathbf{x}, t) \delta(t-\tau)$, then

$$
\left(\frac{\partial \varphi_{\tau}}{\partial t}, \chi\right)_{H_{\eta}}=\int(s-t) e^{-\frac{1}{2}(\mathbf{x}-\mathbf{y})^{2}+\frac{1}{2}(t-s)^{2}} \varphi_{\tau}(\mathbf{x}, t) \bar{\chi}(\mathbf{y}, s) d \mathbf{x} d t d \mathbf{y} d s
$$


which is zero because of the delta functions in $\varphi_{\tau}$ and $\chi$. The orthogonality of $\frac{\partial \varphi_{\tau}}{\partial t}$ and $\chi$ in the $\widetilde{H}$ and $H_{T}$-inner products is verified in a similar way. For example,

$$
\left(\frac{\partial \varphi_{\tau}}{\partial t}, \chi\right)_{H_{T}}=\int(2 \tau-t-s) e^{-\frac{1}{2}(\mathbf{x}-\mathbf{y})^{2}+\frac{1}{2}(t+s-2 \tau)^{2}} \varphi_{\tau}(\mathbf{x}, t) \bar{\chi}(\mathbf{y}, s) d \mathbf{x} d t d \mathbf{y} d s=0
$$

As a result, the components $-i \widehat{h} \varphi_{\tau},-\frac{\partial \varphi_{\tau}}{\partial t}$ of the velocity vector $\frac{d \varphi_{\tau}}{d \tau}$ are orthogonal in all three inner products.

The identification map $I$ in theorem 7 identifies the "horizontal" component $-i \widehat{h} \varphi_{\tau} \in H_{\tau}$ of the velocity $\frac{d \varphi_{\tau}}{d \tau}$ with the vector $-i \widehat{h} \psi(\mathbf{x}, \tau)=\frac{\partial \psi(\mathbf{x}, \tau)}{d \tau}$. In other words, the ordinary Schrödinger evolution is recovered from the evolution $\varphi_{\tau}$ in the space $H$ of functions of four variables by projecting the path $\varphi_{\tau}$ onto the "co-moving" subspace $H_{\tau}$ identified via $I$ with $\mathbf{H} \approx L_{2}\left(\mathbb{R}^{3}\right)$. The orthogonal ("vertical") component of the velocity is due to the motion of $H_{\tau}$ itself.

Note that the equation

$$
\frac{\partial \phi_{\tau}}{\partial \tau}=\left(-\frac{\partial}{\partial t}-i \widehat{h}\right) \phi_{\tau}
$$

in theorem 8 is well known in connection with the relativistic quantum theory and its non-relativistic limit. Indeed, if $\widehat{h}$ is the usual Hamiltonian of the Schrödinger theory, then (18) is the non-relativistic limit of Stueckelberg-Schrödinger equation in the theory of Stueckelberg Ref.[13] and Horwitz \& Piron Ref.[14] (see Ref.[9] for references to later work). An equation of this form is used in Floquet theory that deals with Hamiltonians periodic in time Ref.[15]. It was also suggested by Howland Ref.[16] for treating problems with time-dependent Hamiltonians. One of the advantages of equations of the form (18) is that they can be made relativistic invariant by using a Lorentz invariant operator on the right. Such is the case of the Stueckelberg relativistic theory itself. This theory does treat space and time symmetrically, predicting in particular interference in time Ref.[17]. The latter effect was recently observed Ref.[8] and found to be in a good agreement with the theory Refs.[9]. Another way of making (18) relativistic invariant is by identifying $\widehat{h}$ with the Hamiltonian of the Dirac theory acting on $\mathbb{C}^{4}$-valued functions.

To recover the non-relativistic limit of the Stueckelberg theory Horwitz and Rotbart Ref.[18] use localization around a definite mass (the theory is not on mass shell) as the speed of light $c$ approaches infinity. This yields an approximate equality of the time variable $t$ with the evolution parameter $\tau$. An opposite, more axiomatic approach is taken here. Theorem 8 begins by imposing the condition $t=\tau$ by reverting to the class of functions of the form $\psi(x, t) \delta(t-\tau)$. In doing so one of course loses a possible dispersion of $t$ around $\tau$ and the resulting additional degree of freedom discovered in Ref.[18]. As a trade off, on the chosen class of functions equation (18) becomes equivalent to the Schrödinger equation.

The delta factor in the functions in the family of spaces $H_{\tau}$ is responsible for suppressing the effect of interference in time that is present for more general elements of $H$. Indeed, as follows from the theorem 7 , the norm of a superposition

$$
\psi_{1}(\mathbf{x}, t) \delta(t-\tau)+\psi_{2}(\mathbf{x}, t) \delta(t-\tau)
$$


of functions in $H_{\tau}$ in either $H_{\eta}, \widetilde{H}$, or $H_{T}$-metrics is equal to the norm of the sum

$$
\psi_{1}(\mathbf{x}, \tau)+\psi_{2}(\mathbf{x}, \tau)
$$

in the space $\mathbf{H}$ of functions of three variables $\mathbf{x}$. This is because the additional integration in the time variable is removed by the delta factors. Because there is no integration in time, the interference in time is absent. Furthermore, by theorem 2 the norm of the superposition (20) in $\mathbf{H}$ may be well approximated by $\left\|\psi_{1}(\mathbf{x}, \tau)+\psi_{2}(\mathbf{x}, \tau)\right\|_{L_{2}}$, which ensures the usual interference effects in the space variables. Note also that in the process of integration in time, the time variable in the functions is replaced with the parameter $\tau$. As a result, the evolution parameter $\tau$ gets identified with time.

Furthermore, theorem 3 demonstrates how this particular subset of functions in $H$ may have been physically selected. In fact, recall that $H$ consists of the functions $e^{-t^{2}} \varphi(\mathbf{x}, t)$, where $\varphi \in \widetilde{H}$ while the space $\widetilde{H}$ is well approximated by the space $L_{2}\left(\mathbb{R}^{4}\right)$ of Lebesgue square-integrable functions on $\mathbb{R}^{4}$. The choice of $H$ was dictated by the need to identify Minkowski space with an isometrically embedded submanifold $M_{4} \subset H$. Likewise, the space $H_{T}$ of the time co-moving representation consists of the functions $e^{-(t-\tau)^{2}} \varphi(\mathbf{x}, t)$, with $\varphi \in \widetilde{H} \approx L_{2}\left(\mathbb{R}^{4}\right)$. The variables $(\mathbf{x}, t)$ play a symmetric role in the definition of $L_{2}\left(\mathbb{R}^{4}\right)$, while the factor $e^{-(t-\tau)^{2}}$ breaks this symmetry, making a typical element of $H_{T}$ well localized in the time variable. The fact that under the condition $L=1$ in (2) the factor $e^{-(t-\tau)^{2}}$ falls off to almost zero within a few Planck lengths of $t-\tau$ signifies that for all practical purposes it can be replaced with the delta function $\delta(t-\tau)$. This yields the set of functions in the family of spaces $H_{\tau}$, which by theorems 7 and 8 allows for the usual formalism of quantum mechanics.

Consistency with quantum mechanics imposes an upper bound on the width of the kernel of the metric on $\mathbf{H}$, i.e., the constant $1 / L^{2}$ in (4) and the resulting constant that will appear in (5). The fact that substitution of the delta function in place of the Gaussian factor in the states $e^{-(t-\tau)^{2}} \varphi(\mathbf{x}, t)$ destroys the effect of interference in time imposes a lower bound on the width of the kernel of the metric. Indeed, if the width is too small, the effect observed in Ref.[8] would be lost. Besides the width, the effect of interference also depends on a particular form of the factor $\varphi$ in the superposing states. The resulting estimates, their physical consequences and comparison with the analysis of Refs.[9],[17] will be presented elsewhere.

Newton and Wigner obtained widely known results that conclude that delta functions do not represent spatially localized states in relativistic theory Ref.[19]. Their results challenge the validity of the approach taken here. To investigate the situation note that the results of Ref.[19] were obtained under the following assumptions: (a) the states localized at $t=0$ at the origin form a linear set; (b) this set is invariant under rotations about the origin and reflections of both space and time coordinates; (c) a spatial displacement of a localized state gives an orthogonal state; (d) some regularity conditions ensuring that the generators of the Lorentz group are well defined on the localized states are in place. The orthogonality condition (c) is essential for their derivation. However, in the setting of theorem 6 this 
condition is not true for the delta states and therefore is not likely to remain valid in the theory. If the orthogonality condition is dropped, the remaining conditions are not sufficient to determine the form of localized states in a unique fashion. For instance, the functions $\delta_{a}^{4}$ in $H$ satisfy these conditions (the regularity conditions are satisfied because derivatives of delta functions are in $H$ ). Newton and Wigner give additional reasons why the delta functions $\delta_{a}^{4}$ are not satisfactory localized states of a spinless particle in the standard theory. First of all, they are not square integrable. Also, if a sequence of square integrable functions converges to $\delta_{a}^{4}$ in one frame, it will not do so in general in a Lorentz transformed frame. This argument also loses ground in the setting of theorem 6 . The delta functions have a finite norm in $H$ and in any space $H^{\prime}$ in the theorem. Furthermore, if a sequence of functions approaches $\delta_{a}^{4}$ in one frame, then, as follows from the diagram (13), the Lorentz transformed sequence will approach the same limit in the space $H^{\prime}$ of the frame $K^{\prime}$. The operator having $\delta_{a}^{4}$ as an eigenfunction with the eigenvalue $a^{\mu}$ is the operator $\widehat{x^{\mu}}$ of multiplication by the variable $x^{\mu}, \mu=0,1,2,3$. This operator coincides with the four-point position operator of Horwitz and Rotbart Ref.[18]. Unlike the position operator defined in Ref.[19], this operator has a clear covariant meaning. Indeed, under a Poincaré transformation $\Pi$ on functions, the operator $\widehat{x^{\mu}}$ is transformed via

$$
\widehat{x^{\mu}} \longrightarrow \delta_{\Pi}^{-1} \widehat{x^{\mu}} \delta_{\Pi}
$$

Therefore the kernel of the transformed operator is given by

$$
\int \delta^{4}(\Pi x-u) u^{\mu} \delta^{4}(u-v) \delta^{4}\left(\Pi^{-1} v-y\right) d u d v=(\Pi x)^{\mu} \delta^{4}(\Pi x-\Pi y),
$$

which yields the operator $\widehat{x^{\prime \mu}}$ of multiplication by $x^{\prime \mu}=(\Pi x)^{\mu}$. The covariant property of the states $\delta_{a}^{4}$ and the operator $\widehat{x^{\mu}}$ does not mean by itself that the found objects are physical. Indeed, the state $\delta_{a}^{4}$ does not correspond to a particle of a given mass (it is not "on the shell"). Also, the presence of the delta factor $\delta_{\mathbf{a}}^{3}$ signifies that the wave packet $\delta_{a}^{4}$ contains negative energy components. Furthermore, as shown in Ref.[20], a state function having a compact support at a certain time and evolving in accordance with the usual relativistic equations will evolve out of the light cone. Although these problems are typical in a relativistic theory and were understood within the Stueckelberg approach by making the mass of a particle a dynamical variable Ref.[14], they must be reexamined in the new setting.

To complete the embedding of the standard quantum mechanical formalism into the new geometric framework it remains to demonstrate that the action of unitary transformations on $L_{2}\left(\mathbb{R}^{3}\right)$ can be realized by isomorphisms of $H$. The following theorem proves just that.

Theorem 9. An arbitrary isomorphism $V: H \longrightarrow H^{\prime}$ of Hilbert spaces that preserves the indefinite metric on $H$ and maps subspaces $H_{\tau}$ of functions $\psi(x, t) \delta(t-\tau)$ onto themselves yields a family of unitary transformations on the space $\mathbf{H} \approx L_{2}\left(\mathbb{R}^{3}\right)$ of functions $\psi(x, t)$. Moreover, all unitary transformations on $\mathbf{H}$ are obtained in such a way.

Proof. Let $V$ be an isomorphism in the statement of the theorem. Consider its action on the subspace $H_{\tau_{1}}$ of all functions $\varphi_{\tau_{1}}(\mathbf{x}, t)=\psi(\mathbf{x}, t) \delta\left(t-\tau_{1}\right)$ in $H$ for some fixed $\tau_{1}$. By assumption $V$ maps $H_{\tau_{1}}$ onto the subspace $H_{\tau_{2}} \subset H$ 
of all functions $\varphi_{\tau_{2}}(\mathbf{x}, t)=\psi(\mathbf{x}, t) \delta\left(t-\tau_{2}\right)$ for some $\tau_{2}$. So, $V\left(\varphi_{\tau_{1}}\right)=U_{\tau_{2}, \tau_{1}}(\psi) \delta\left(t-\tau_{2}\right)$ for some operator $U_{\tau_{2}, \tau_{1}}$ on the space $\mathbf{H}$. By theorem 7 the indefinite norm of $V\left(\varphi_{\tau_{1}}\right)$ in $H$ is equal to the norm of $U_{\tau_{2}, \tau_{1}}(\psi)$ in $\mathbf{H}$. Because $V$ is norm preserving, so is $U_{\tau_{2}, \tau_{1}}$. Also, because $V\left(H_{\tau_{1}}\right)=H_{\tau_{2}}$ the image of $U_{\tau_{2}, \tau_{1}}$ must be the entire space $\mathbf{H}$. It follows that for all values $\tau_{1} \in \mathbb{R}$ the operator $U_{\tau_{2}, \tau_{1}}$ is unitary on $\mathbf{H}$.

Now, given a unitary transformation $U$ on $\mathbf{H}$, define a transformation $V$ on functions $\varphi_{\tau}(\mathbf{x}, t)=\psi(\mathbf{x}, t) \delta(t-\tau)$, $\psi \in \mathbf{H}, \tau \in \mathbb{R}$ via $V\left(\varphi_{\tau}\right)=U(\psi) \delta(t-\tau)$. Because $U$ is unitary and the delta function $\delta(t-\tau)$ removes integration in time, $V$ preserves the indefinite and the Hilbert norm of the functions $\varphi_{\tau}$ for all $\tau \in \mathbb{R}$. The set $S=\cup_{\tau} H_{\tau}$ of all functions $\varphi_{\tau}, \tau \in \mathbb{R}$ is complete in $H$. It follows that the linear extension $W$ of $V$ to $H$ exists and preserves the indefinite and the Hilbert norms of all functions in $H$. Because $V\left(H_{\tau}\right)=H_{\tau}$, one has $V(S)=S$. Since $S$ is complete in $H$, the range of $W$ must be dense in $H$. It follows that $W$ is an automorphism of $H$ that preserves the indefinite metric.

\section{CONCLUSION}

The proved theorems demonstrate that there exists a nice covariant geometric framework that unifies the mathematical formalism of classical relativity and quantum mechanics. Since these two have always been in some tension, the existence of such a framework is at the same time surprising and promising. In addition to the standard results of classical relativity and quantum theory the unifying geometric framework addresses the observed asymmetry of space and time in quantum mechanics and the related nonappearance of quantum interference in time in the nonrelativistic domain. It also explains why the evolution parameter in relativistic quantum theories does not reveal itself in non-relativistic experiments.

Theorem 6 is the crux of the proposed unification scheme. It demonstrates that different inertial frames in the framework define different functional realizations of the abstract Hilbert space of states. Consequently, the Hilbert space of state functions is coordinate dependent and therefore unphysical. Instead, the abstract Hilbert space $\mathbf{S}$ acquires the status of a physical space of states. Only in some situations a single realization of $\mathbf{S}$ suffices, in which case it can be identified with $\mathbf{S}$ and becomes physically meaningful. The struggle to resolve difficulties of the unification of relativity and quantum mechanics may be due in part to a failure of recognizing this fact.

Furthermore, theorem 6 proves that the invariant geometric properties of Minkowski space, being at the heart of Einstein relativity, are particular realizations of geometric properties of the space $\mathbf{S}$. The embedding $\omega$ in the theorem allows one to consider the Poincaré transformations on $N$ as isomorphisms of Hilbert spaces of functions. More generally, this remains true for arbitrary transformations on $N$. Likewise, tensor fields on $N$ are realized as elements of the tensor algebra of $\mathbf{S}$. In addition to providing a possible functional origin of relativity on space-time, this result may explain why quantum mechanics is a linear theory. In simple terms, mathematical objects of a non-linear nature 
on the space-time become linear when extended to $\mathbf{S}$.

The obtained results do not mean, of course, that the issue of unification of relativity and quantum is resolved. Rather, the main outcome of the paper is a new mathematical structure that provides a possible formalism for the unification. The fact that at first glance this structure is in agreement with special relativity and quantum theory is very encouraging and should not be underestimated. However, it remains to be seen if this mathematical structure may serve a basis for a sound physical framework capable of resolving the problem.

\section{ACKNOWLEDGMENTS}

It is a pleasure to thank Malcolm Forster for valuable discussions, especially those related to theorem 6 , for suggesting a way to streamline the proof of theorem 8 and for numerous suggestions that helped improve the paper. I am indebted to Larry Horwitz for critical review of the manuscript, useful suggestions and references that affected the paper in a significant way and for a fruitful discussion of the issues of relativistic quantum mechanics. I would also like to thank participants of the International Conference on Classical and Quantum Relativistic Dynamics of Particles and Fields (2008), where this paper was presented for their sincere interest and great questions.

[1] I.M. Gel'fand and N.Y. Vilenkin, Generalized Functions, Vol.4, Academic Press, New York and London, (1964)

[2] A. Kryukov, Int. J. Math. \& Math. Sci. 14, 2241 (2005)

[3] A. Kryukov, Found. Phys. 34, 1225 (2004)

[4] A. Kryukov (unpublished)

[5] A. Kryukov, Found. Phys. 36, 175 (2006)

[6] A. Kryukov, Found. Phys. 37, 3 (2007)

[7] A. Kryukov, Phys. Lett. A 370, 419 (2007)

[8] F. Linder et al, Phys. Rev. Lett. 95, 040401 (2005)

[9] L.P. Horwitz Phys. Lett. A 355, 1 (2006)

[10] I.M. Gel'fand and A.N. Kolmogorov, Dokl. Akad. Nauk SSSR, 2211 (1939)

[11] I. Segal, Illinois J. Math. 6, 500 (1962)

[12] V. Bargmann, Proc. Nat. Academy Sci. U.S.A. 48, 199 (1962)

[13] E.C.G. Stueckelberg, Helv. Phys. Acta 14, 372, 585 (1941); Helv. Phys. Acta 15, 23 (1942)

[14] L.P. Horwitz and C. Piron Helv. Phys. Acta 46, 316 (1973)

[15] H.L. Cycon, R.G. Froese, W. Kirsch, and B. Simon, Schrödinger Operators with Application to Quantum Mechanics and Global Geometry, Springer- Verlag, New York (1987)

[16] J.S. Howland, Indiana Univ. Math. Jour. 28, 471 (1979)

[17] L.P. Horwitz and Y. Rabin Lett. Nuovo Cimento 17, 501 (1976) 
[18] L.P. Horwitz and F. Rotbart, Phys. Rev. D 24, 2127 (1981)

[19] T.D. Newton and E.P. Wigner, Reviews of Modern Physics, 21400 (1949)

[20] G.C. Hegerfeldt Phys. Rev. D 10, 3320 (1974) 